\title{
Synthesis of Novel Quinaldine Based Squaraine Dyes: Effect of Substituents and Role of Electronic Factors
}

\author{
Kuthanapillil Jyothish, Kalliat T. Arun and Danaboyina Ramaiah*
}

Photosciences and Photonics Division

Regional Research Laboratory (CSIR)

Trivandrum 695 019, INDIA

*To whom correspondence should be addressed at:

Photosciences and Photonics Division

Regional Research Laboratory (CSIR)

Trivandrum 695 019, INDIA.

Tel: +91 471 2515362. Fax: +91 $4712490186,2491712$.

E-mail : $\underline{\text { d_ramaiah@rediffmail.com or rama@ csrrltrd.ren.nic.in }}$

\section{Supporting Information}

General procedure for the synthesis of quinaldines, quinaldinium salts, semisquaraines and squaraine dyes, Figures S1-S3 showing the progress of the condensation reaction between squaric acid and the quinaldinium salts $\mathbf{1 b}, \mathbf{1 c}$ and $\mathbf{1 e}$ by absorption spectroscopy and Figures S4-S7 showing the ${ }^{1} \mathrm{H}$ and ${ }^{13} \mathrm{C}$ NMR spectra of the representative semisquaraines and the squaraine dyes. 


\section{General methods}

All melting points are uncorrected and were determined on a Mel-Temp II melting point apparatus. The IR spectra were recorded on a Perkin Elmer Model 882 infrared spectrometer. The electronic absorption spectra were recorded on a Shimadzu UV-3101 or 2401 PC UV-VISNIR scanning spectrophotometer. All the solvents used were purified and distilled before use. The progress of the reaction was monitored by recording absorption spectrum of the reaction mixture at various time intervals. For this $25 \mu \mathrm{L}$ of the reaction mixture was taken out and diluted to $3 \mathrm{~mL}$ with distilled n-butanol and the spectra recorded.

Starting materials. 4-Aminophenol (mp 188-189 $\left.{ }^{0} \mathrm{C}\right),{ }^{1}$ 4-ethoxyaniline (bp 250-251 $\left.{ }^{0} \mathrm{C}\right),{ }^{1}$ 2-bromo-4-nitrophenol (mp 184-185 $\left.{ }^{0} \mathrm{C}\right),{ }^{1}$ 4-iodoaniline $\left(\mathrm{mp} 63-64{ }^{0} \mathrm{C}\right),{ }^{1}$ 4-bromoaniline $(\mathrm{mp} 61$ $\left.62{ }^{0} \mathrm{C}\right),{ }^{1} 4$-nitroaniline, $\left(\mathrm{mp} 148-149{ }^{0} \mathrm{C}\right),{ }^{1}$ 6-hydroxy-2-quinaldine (mp 262-264 $\left.{ }^{0} \mathrm{C}\right),{ }^{2}$ 6-ethoxy2-quinaldine (mp 71-72 $\left.{ }^{0} \mathrm{C}\right),{ }^{2}$ 6-hydroxy-7-bromo-2-quinaldine (mp 235-237 $\left.{ }^{0} \mathrm{C}\right)$, 6-iodo-2quinaldine (mp 108-109 $\left.{ }^{0} \mathrm{C}\right){ }^{3}$ 6-bromo-2-quinaldine (mp 95-96 $\left.{ }^{0} \mathrm{C}\right),{ }^{3}$ 6-nitro-2-quinaldine (mp 165-166 $\left.{ }^{0} \mathrm{C}\right),{ }^{4}$ 6-hydroxy-N-methyl-2-quinaldinium iodide (1a) (mp 238-239 $\left.{ }^{0} \mathrm{C}\right),{ }^{2}$ 6-ethoxy-Nmethyl-2-quinaldinium iodide (1b) (mp 182-183 $\left.{ }^{0} \mathrm{C}\right),{ }^{2} \mathrm{~N}$-methyl-2-quinaldinium iodide (1d) (mp 195-196 $\left.{ }^{0} \mathrm{C}\right),{ }^{5}$ 6-iodo-N-methyl-2-quinaldinium iodide (1e) (mp 222-223 $\left.{ }^{0} \mathrm{C}\right),{ }^{3}$ 6-bromo-Nmethyl-2-quinaldinium iodide (1f) $\left(\mathrm{mp} 237{ }^{0} \mathrm{C}\right),{ }^{3}$ 6-nitro-N-methyl-2-quinaldinium iodide (1g) $\left(\mathrm{mp} 214-215{ }^{0} \mathrm{C}\right),{ }^{4}$ and the squaraine dye $(\mathbf{3 d})\left(\mathrm{mp} 298{ }^{0} \mathrm{C}\right),{ }^{6}$ were prepared by modifying the reported procedures. 2-Methylquinoline was purchased from Aldrich and used as such while squaric acid was a gift from Professor Waldemar Adam, University of Würzburg, Germany.

\section{General procedure for the preparation of quinaldines ${ }^{7}$}

To a refluxing solution of the corresponding aniline derivative $(18 \mathrm{mmol})$ in $6 \mathrm{~N} \mathrm{HCl}(25$ $\mathrm{mL})$, crotonaldehyde $(72 \mathrm{mmol})$ was added drop wise over a period of $1 \mathrm{~h}$ and refluxed for $6 \mathrm{~h}$. 
The reaction mixture was cooled to room temperature, made alkaline using ammonia solution and was then extracted with diethyl ether. Removal of the solvent under reduced pressure gave a residue, which was subjected to column chromatography over silica gel. Elution of the column with a mixture of ethyl acetate and petroleum ether (1:9) gave the corresponding quinaldine derivatives in good yields.

Preparation of 6-cyano-2-quinaldine. A mixture of 6-bromo-2-methylquinoline (600 mg, $2.7 \mathrm{mmol}$ ), cuprous cyanide (400 $\mathrm{mg}, 4.5 \mathrm{mmol})$ and pyridine $(6 \mathrm{~mL})$ was heated in a sealed tube at $200{ }^{0} \mathrm{C}$ for $24 \mathrm{~h}$. The deep brown solution was shaken with aqueous ammonia and dichloromethane and the organic layer was washed with water. Removal of the solvent gave a residue which was then subjected to column chromatography over silica gel. Elution of the column with a mixture of ethyl acetate and petroleum ether (1:9) gave $75 \%$ of the quinaldine derivative, mp 168-170 ${ }^{0} \mathrm{C}$, IR $(\mathrm{KBr}) v_{\max } 3031,2224,1619 \mathrm{~cm}^{-1} ;{ }^{1} \mathrm{H}-\mathrm{NMR}\left(\mathrm{CDCl}_{3}\right) \delta 8.17$ $(1 \mathrm{H}, \mathrm{s}), 8.10(2 \mathrm{H}, \mathrm{dd}, \mathrm{J}=11.38 \mathrm{~Hz}), 7.80(1 \mathrm{H}, \mathrm{d}, \mathrm{J}=8.7 \mathrm{~Hz}), 7.40(1 \mathrm{H}, \mathrm{d}, \mathrm{J}=8.46 \mathrm{~Hz}), 2.79$ $(3 \mathrm{H}, \mathrm{s}) ;{ }^{13} \mathrm{C}$ NMR $\left(\mathrm{DMSO}_{-} \mathrm{d}_{6}\right) \delta 162.5,148.8,136.2,133.7,130.2,130.1,125.8,123.7,118.6$, 109.3, 25.6.

\section{General procedure for the preparation of quinaldinium salts 1a-h}

A mixture of the corresponding quinaldine $(1 \mathrm{mmol})$ and methyl iodide $(4 \mathrm{mmol})$ was heated in a sealed tube at $100-105{ }^{\circ} \mathrm{C}$ for $12 \mathrm{~h}$. The precipitate formed was filtered, washed thoroughly with cold diethyl ether and subjected to column chromatography over silica gel. Elution of the column with a mixture of methanol and chloroform (1:4) gave the corresponding quinaldinium salts 1a-h in good yields. 1c: $\left(55 \%, \mathrm{mp} 296-297{ }^{0} \mathrm{C}\right),{ }^{1} \mathrm{H}-\mathrm{NMR}$ (DMSO-d 6 ) $\delta 8.68$ $(1 \mathrm{H}, \mathrm{s}), 8.61(1 \mathrm{H}, \mathrm{d}, \mathrm{J}=8.5 \mathrm{~Hz}), 7.76(1 \mathrm{H}, \mathrm{d}, \mathrm{J}=8.5 \mathrm{~Hz}), 7.28(1 \mathrm{H}, \mathrm{s}), 4.24(3 \mathrm{H}, \mathrm{s}), 2.91(3 \mathrm{H}, \mathrm{s})$; ${ }^{13}$ C NMR (DMSO-d 6 ) $\delta 159.4,154.4,142.0,131.3,129.9,126.8,124.2,122.5,109.9,38.6,22.1$. 
1h: $\left(75 \%, \mathrm{mp} 202-204^{0} \mathrm{C}\right)$, IR (KBr) $v_{\max } 3065,2986,2233,1625 \mathrm{~cm}^{-1} .{ }^{1} \mathrm{H}-\mathrm{NMR}$ $\left(\right.$ DMSO-d $\left._{6}\right) \delta 9.40(1 \mathrm{H}, \mathrm{s}), 9.32(1 \mathrm{H}, \mathrm{d}, \mathrm{J}=8.52 \mathrm{~Hz}), 8.82(2 \mathrm{H}, \mathrm{d}, \mathrm{J}=9.81 \mathrm{~Hz}), 8.29(1 \mathrm{H}$, $\mathrm{d}, \mathrm{J}=8.7 \mathrm{~Hz}), 4.50(3 \mathrm{H}, \mathrm{s}), 3.1(3 \mathrm{H}, \mathrm{s}) ;{ }^{13} \mathrm{C} \mathrm{NMR}\left(\mathrm{DMSO}_{-} \mathrm{d}_{6}\right) \delta 164.5,145.5,140.6,136.2$ $135.4,127.2,127.0,121.0,117.2,111.5,38.7,23.6$.

General procedure for the synthesis of semisquaraines 2a-c

A mixture of the corresponding quinaldinium salt $(0.06 \mathrm{mmol})$, squaric acid $(0.06 \mathrm{mmol})$ and quinoline $(0.5 \mathrm{~mL})$ was refluxed in a mixture of $n$-butanol and benzene $(6 \mathrm{~mL}$ each, $1: 1)$ with azeotropic distillation of water for $24 \mathrm{~h}$. The solvent was distilled off under reduced pressure to obtain a residue which was chromatographed over silica gel. Elution of the column with a mixture (1:9) of methanol and chloroform gave the semisquaraine derivatives 2a-c in good yields calculated based on conversion.

2a: $95 \%$, mp 150-152 ${ }^{0} \mathrm{C}$, IR (KBr) $v_{\max } 3412,3042,2963,1761,1606 \mathrm{~cm}^{-1} .{ }^{1} \mathrm{H}-\mathrm{NMR}$ $\left(\mathrm{CDCl}_{3}+\mathrm{DMSO}_{-} \mathrm{d}_{6}, 1: 4\right) \delta 10.06(1 \mathrm{H}, \mathrm{OH}), 9.29(1 \mathrm{H}, \mathrm{d}, \mathrm{J}=11.4 \mathrm{~Hz}), 7.89(1 \mathrm{H}, \mathrm{d}, \mathrm{J}=9.3 \mathrm{~Hz})$ $7.73(1 \mathrm{H}, \mathrm{d}, \mathrm{J}=9.4 \mathrm{~Hz}), 7.34(1 \mathrm{H}, \mathrm{d}, \mathrm{J}=9.3 \mathrm{~Hz}), 7.17(\mathrm{IH}, \mathrm{s}), 6.14(1 \mathrm{H}, \mathrm{s}), 4.64(2 \mathrm{H}, \mathrm{t}, \mathrm{J}=6.5$

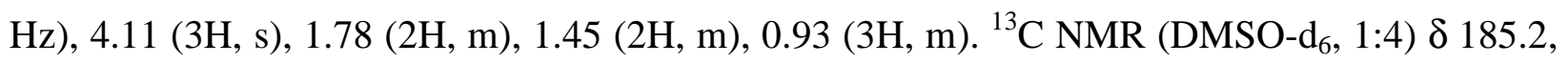
177.2, 175.4, 171.5, 155.8, 152.5, 137.4,132.8, 127.1, 124.5, 123.0, 119.4, 111.5, 95.6, 70.8, 37.8, 31.7, 18.2, 13.5; FAB-MS: $\mathrm{m} / \mathrm{z}=325.140\left(\right.$ calcd 325.139 for $\left.\mathrm{C}_{19} \mathrm{H}_{19} \mathrm{NO}_{4}\right)$.

2b: $90 \%$, mp 184-186 ${ }^{\circ} \mathrm{C}$, IR (KBr) $v_{\max } 3016,2923,1756,1601 \mathrm{~cm}^{-1} .{ }^{1} \mathrm{H}-\mathrm{NMR}\left(\mathrm{CDCl}_{3}\right) \delta 9.41$ $(1 \mathrm{H}, \mathrm{d}, \mathrm{J}=9.3 \mathrm{~Hz}), 7.84(\mathrm{IH}, \mathrm{d}, \mathrm{J}=9.3 \mathrm{~Hz}), 7.63(1 \mathrm{H}, \mathrm{d}, \mathrm{J}=9.4 \mathrm{~Hz}), 7.33(1 \mathrm{H}, \mathrm{d}, \mathrm{J}=9.5 \mathrm{~Hz})$ $7.09(1 \mathrm{H}, \mathrm{s}), 6.15(1 \mathrm{H}, \mathrm{s}), 4.71(2 \mathrm{H}, \mathrm{t}, \mathrm{J}=6.6 \mathrm{~Hz}), 4.14(2 \mathrm{H}, \mathrm{t}, \mathrm{J}=6.9 \mathrm{~Hz}), 4.05(3 \mathrm{H}, \mathrm{s}), 1.78$ $(5 \mathrm{H}, \mathrm{m}), 1.49(2 \mathrm{H}, \mathrm{m}), 0.96(3 \mathrm{H}, \mathrm{m}) .{ }^{13} \mathrm{C} \mathrm{NMR}\left(\mathrm{CDCl}_{3}\right) \delta 184.1,176.3,174.3,170.2,156.6$, 153.3, 136.7, 126.6, 125.6, 122.8, 117.4, 109.5, 94.6, 71.9, 63.9, 37.1, 31.8, 18.2, 14.2, 13.5, 13.3; FAB-MS: $\mathrm{m} / \mathrm{z}=353.164$ (calcd 353.163 for $\mathrm{C}_{21} \mathrm{H}_{23} \mathrm{NO}_{4}$ ). 
2c: $90 \%, \mathrm{mp} 182-184{ }^{0} \mathrm{C}$, IR (KBr) $v_{\max } \quad 3421, \quad 2950, \quad 1760,1589 \mathrm{~cm}^{-1} .{ }^{1} \mathrm{H}-\mathrm{NMR}$ $\left(\mathrm{CDCl}_{3}+\mathrm{DMSO}_{6}, 1: 4\right) \delta 9.31(1 \mathrm{H}, \mathrm{d}, \mathrm{J}=9.2 \mathrm{~Hz}), 8.12(1 \mathrm{H}, \mathrm{s}), 7.88(1 \mathrm{H}, \mathrm{d}, \mathrm{J}=9.2 \mathrm{~Hz}), 7.3$ $(1 \mathrm{H}, \mathrm{s}), 6.15(1 \mathrm{H}, \mathrm{s}), 4.65(2 \mathrm{H}, \mathrm{t}, \mathrm{J}=6.7 \mathrm{~Hz}), 4.09(3 \mathrm{H}, \mathrm{s}), 1.78(2 \mathrm{H}, \mathrm{m}), 1.48(2 \mathrm{H}, \mathrm{m}), 0.96(3 \mathrm{H}$, m). ${ }^{13} \mathrm{C}$ NMR (DMSO-d 6 , 1:4) $\delta 185.8,177.3,175.4,172.0,156.2,136.2,133.3,126.1,124.5$, 123.2, 118.0, 111.4, 97.8, 71.8, 38.8, 31.5, 18.1, 13.3; FAB-MS: $\mathrm{m} / \mathrm{z}=404.256($ calcd 404.255 for $\left.\mathrm{C}_{19} \mathrm{H}_{18} \mathrm{BrNO}_{4}\right)$.

\section{General procedure for the synthesis of squaraine dyes 3d-f}

A mixture of the corresponding quinaldinium salt $(0.06 \mathrm{mmol})$, and squaric acid $(0.03$ mmol) and quinoline $(0.5 \mathrm{~mL})$ was refluxed in a mixture of $n$-butanol and benzene $(6 \mathrm{~mL}$ each, 1:1) with azeotropic distillation of water for $24 \mathrm{~h}$. The solvent was distilled off under reduced pressure to obtain a residue which was chromatographed over silica gel. Elution of the column with a mixture (1:9) of methanol and chloroform gave the corresponding squaraine dyes $\mathbf{3 d - f}$ in good yields calculated based on the conversion.

3e: $85 \%$, mp 314-315 ${ }^{0} \mathrm{C}$, IR (KBr) $v_{\max } 2962,1620,1553 \mathrm{~cm}^{-1} .{ }^{1} \mathrm{H}-\mathrm{NMR}$ (DMSO-d $\left.\mathrm{d}_{6}\right) \delta 9.23$ $(1 \mathrm{H}, \mathrm{d}, \mathrm{J}=9.4 \mathrm{~Hz}), 9.19(1 \mathrm{H}, \mathrm{d}, \mathrm{J}=9.2 \mathrm{~Hz}), 7.1-8.05(8 \mathrm{H}, \mathrm{m}$, aromatic), $5.89(1 \mathrm{H}, \mathrm{s}$, vinylic), $5.72(1 \mathrm{H}, \mathrm{s}$, vinylic), $3.81(3 \mathrm{H}, \mathrm{s}), 3.71(3 \mathrm{H}, \mathrm{s})$; FAB-MS: $\mathrm{m} / \mathrm{z}=643.944$ (calcd 643.946 for $\mathrm{C}_{26} \mathrm{H}_{18} \mathrm{I}_{2} \mathrm{~N}_{2} \mathrm{O}_{2}$ ).

3f: $86 \%, \mathrm{mp} 336-338{ }^{0} \mathrm{C}$, IR (KBr) $v_{\max } 3056,1613,1580 \mathrm{~cm}^{-1} .{ }^{1} \mathrm{H}-\mathrm{NMR}$ (DMSO-d $\left.\mathrm{d}_{6}\right) \delta 9.31$ $(1 \mathrm{H}, \mathrm{d}, \mathrm{J}=9.5 \mathrm{~Hz}), 9.28(1 \mathrm{H}, \mathrm{d}, \mathrm{J}=9.3 \mathrm{~Hz}), 7.3-8.4(8 \mathrm{H}, \mathrm{m}$, aromatic $), 5.86(1 \mathrm{H}, \mathrm{s}$, vinylic), 5.78(1H, s, vinylic), $3.85(3 \mathrm{H}, \mathrm{s}) ; 3.78(3 \mathrm{H}, \mathrm{s}) ; \mathrm{FAB}-\mathrm{MS}: \mathrm{m} / \mathrm{z}=550.246$ (calcd 550.248 for $\left.\mathrm{C}_{26} \mathrm{H}_{18} \mathrm{Br}_{2} \mathrm{~N}_{2} \mathrm{O}_{2}\right)$. 


\section{General procedure for the synthesis of squaraine dyes $3 \mathrm{~g}-\mathrm{h}$}

A mixture of the corresponding quinaldinium salt $(0.06 \mathrm{mmol})$, and squaric acid $(0.03$ mmol), tributylorthoformate $(0.5 \mathrm{~mL})$ and quinoline $(0.5 \mathrm{~mL})$ was heated in a mixture of $n$ butanol and benzene $(6 \mathrm{~mL}$ each, $1: 1)$ at $60-70{ }^{0} \mathrm{C}$ for $6 \mathrm{~h}$. The solvent was distilled off under reduced pressure to obtain a residue which was then thoroughly washed with methanol to give the corresponding squaraine dyes $\mathbf{3} \mathbf{~ g - h}$ in good yields (calculated based on conversion).

3g: 86\%, mp 290-292 ${ }^{0} \mathrm{C}$, IR (KBr) $v_{\max }$ 3032, 1616, $1579 \mathrm{~cm}^{-1} .{ }^{1} \mathrm{H}-\mathrm{NMR}\left(\mathrm{DMSO}_{6}\right) \delta 9.49$ $(1 \mathrm{H}, \mathrm{d}, \mathrm{J}=9.4 \mathrm{~Hz}), 9.45(1 \mathrm{H}, \mathrm{d}, \mathrm{J}=9.5 \mathrm{~Hz}), 7.4-8.27(8 \mathrm{H}, \mathrm{m}$, aromatic $), 5.92(1 \mathrm{H}, \mathrm{s}$, vinylic $)$, $5.85(1 \mathrm{H}, \mathrm{s}$, vinylic), $3.92(3 \mathrm{H}, \mathrm{s}), 3.84(3 \mathrm{H}, \mathrm{s})$; FAB-MS: $\mathrm{m} / \mathrm{z}=498.149$ (calcd 498.150 for $\mathrm{C}_{27} \mathrm{H}_{22} \mathrm{~N}_{4} \mathrm{O}_{6}$ ).

3h: 84\%, mp 306-308 ${ }^{0} \mathrm{C}, \mathrm{IR} v_{\max }(\mathrm{KBr}) 3029,2225,1602 \mathrm{~cm}^{-1} ;{ }^{1} \mathrm{H}-\mathrm{NMR}\left(\mathrm{DMSO}-\mathrm{d}_{6}\right) \delta 9.39$ $(1 \mathrm{H}, \mathrm{d}, \mathrm{J}=8.9 \mathrm{~Hz}), 9.42(1 \mathrm{H}, \mathrm{d}, \mathrm{J}=9.4 \mathrm{~Hz}), 7.35-8.28(8 \mathrm{H}, \mathrm{m}$, aromatic $), 5.87(1 \mathrm{H}, \mathrm{s}$, vinylic $)$, $5.79(1 \mathrm{H}, \mathrm{s}$, vinylic), $3.95(3 \mathrm{H}, \mathrm{s}), 3.82(3 \mathrm{H}, \mathrm{s}) ; \mathrm{FAB}-\mathrm{MS}: \mathrm{m} / \mathrm{z}=442.472$ (calcd 442.470 for $\mathrm{C}_{28} \mathrm{H}_{18} \mathrm{Br}_{2} \mathrm{~N}_{4} \mathrm{O}_{2}$ ).

Synthesis of squaraine dye 4. A mixture semisquaraine $2 \mathrm{a}(0.06 \mathrm{mmol})$ and the quinaldinium salt 1e $(0.06 \mathrm{mmol})$, and quinoline $(0.5 \mathrm{~mL})$ was refluxed in a mixture of $n$-butanol and benzene $(6 \mathrm{~mL}$ each, 1:1) for $12 \mathrm{~h}$. The solvent was distilled off under reduced pressure to obtain a residue which was chromatographed over silica gel. Elution of the column with a mixture (1:9) of methanol and chloroform gave the corresponding squaraine dye 4: 96\% (based on conversion), mp $316-318{ }^{0} \mathrm{C}$, IR $(\mathrm{KBr}) v_{\max } 3442,3046,1729,1621,1579,1560 \mathrm{~cm}^{-1} .{ }^{1} \mathrm{H}-$ NMR (DMSO-d $\left.{ }_{6}\right) \delta 10.2(1 \mathrm{H}, \mathrm{OH}), 9.29(1 \mathrm{H}, \mathrm{d}, \mathrm{J}=9.2 \mathrm{~Hz}), 9.01(1 \mathrm{H}, \mathrm{d}, \mathrm{J}=9.5 \mathrm{~Hz}), 7.86-7.01$ $(8 \mathrm{H}, \mathrm{m}), 5.77(1 \mathrm{H}, \mathrm{s}$, vinylic $), 5.44(1 \mathrm{H}, \mathrm{s}$, vinylic $), 3.9(3 \mathrm{H}, \mathrm{s}), 3.5(3 \mathrm{H}, \mathrm{s}) ; \mathrm{FAB}-\mathrm{MS}$ : $\mathrm{m} / \mathrm{z}=534.040\left(\right.$ calcd 534.039 for $\left.\mathrm{C}_{26} \mathrm{H}_{19} \mathrm{IN}_{2} \mathrm{O}_{3}\right)$. 


\section{References}

1) Vogel, A. I. A Textbook of Practical Organic Chemistry, Fourth Edition. ELBS and Longman Group Ltd.: London 1978.

2) Doebner, A.; von Miller, W. Ber. 1884, 17, 708.

3) Jha, B. N.; Banerji, J. C. Dyes and Pigments, 1983, 4, 77.

4) Utermohlen, Jr. W. B. J. Org. Chem. 1943, 8, 544

5) Doebner, A; von Miller, W. Ber., 1883, 16, 2465.

6) Bernstein, J.; Tristani-Kendra, M.; Eckhardt, C. J. J. Phys. Chem. 1986, 90, 1069.

7) (a) Jones, G. Ed. The Chemistry of Heterocyclic compounds, Part I, Interscience Publishers, Great Britain, 1977. (b) Spivey, A. M.; Curd, F. H. S. J. Chem. Soc. 1949, 2656. (c) Leir, C. M. J. Org. Chem. 1977, 42, 911. 


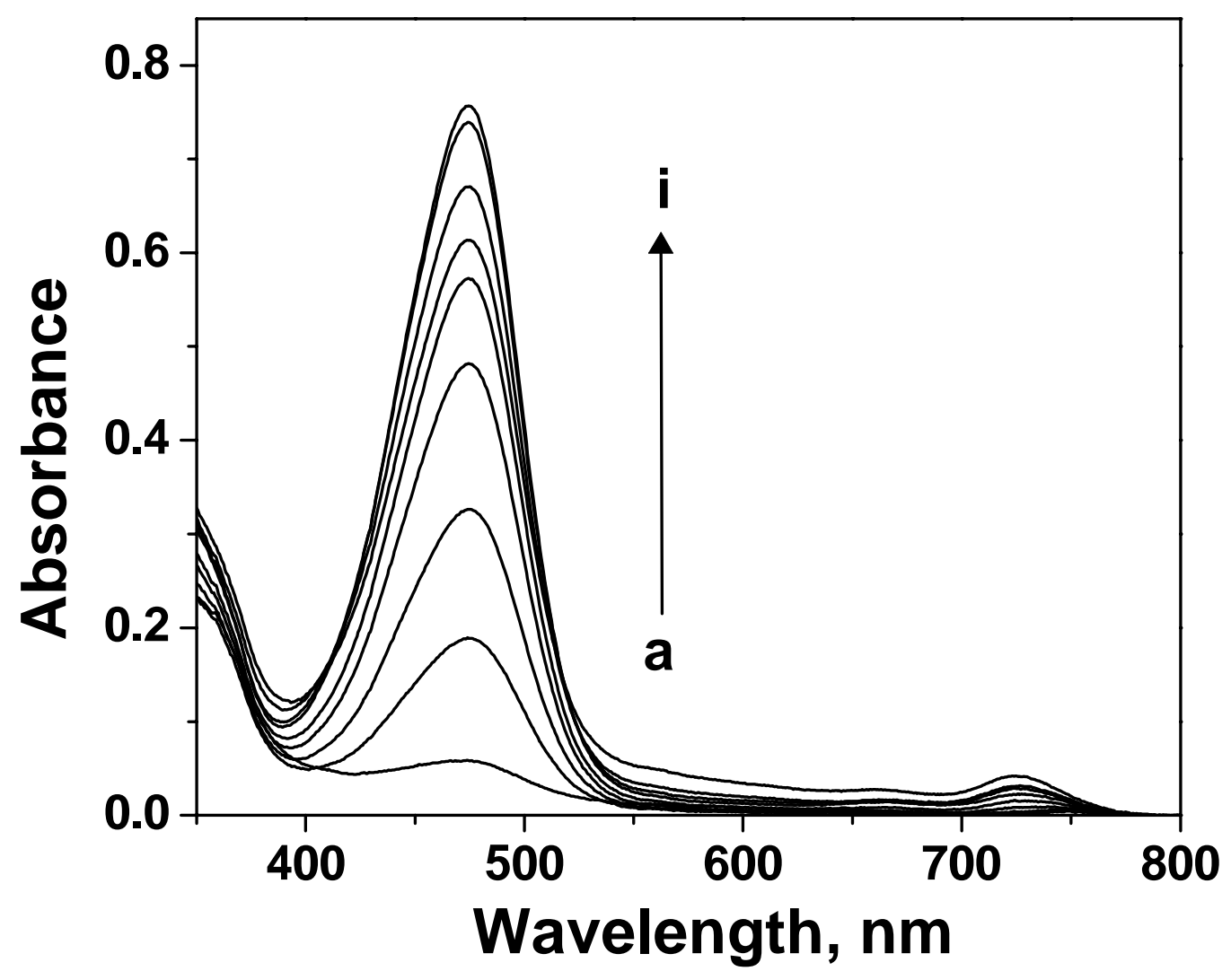

Figure S1. Change in absorption spectra obtained for the reaction between squaric acid and the quinaldinium salt $\mathbf{1 b}$ at various time intervals. Time (a) 1, (b) 2, (c) 3, (d) 4 (e) 5, (f) 7, (g) 10, (h) 13 , and (i) $24 \mathrm{~h}$. 


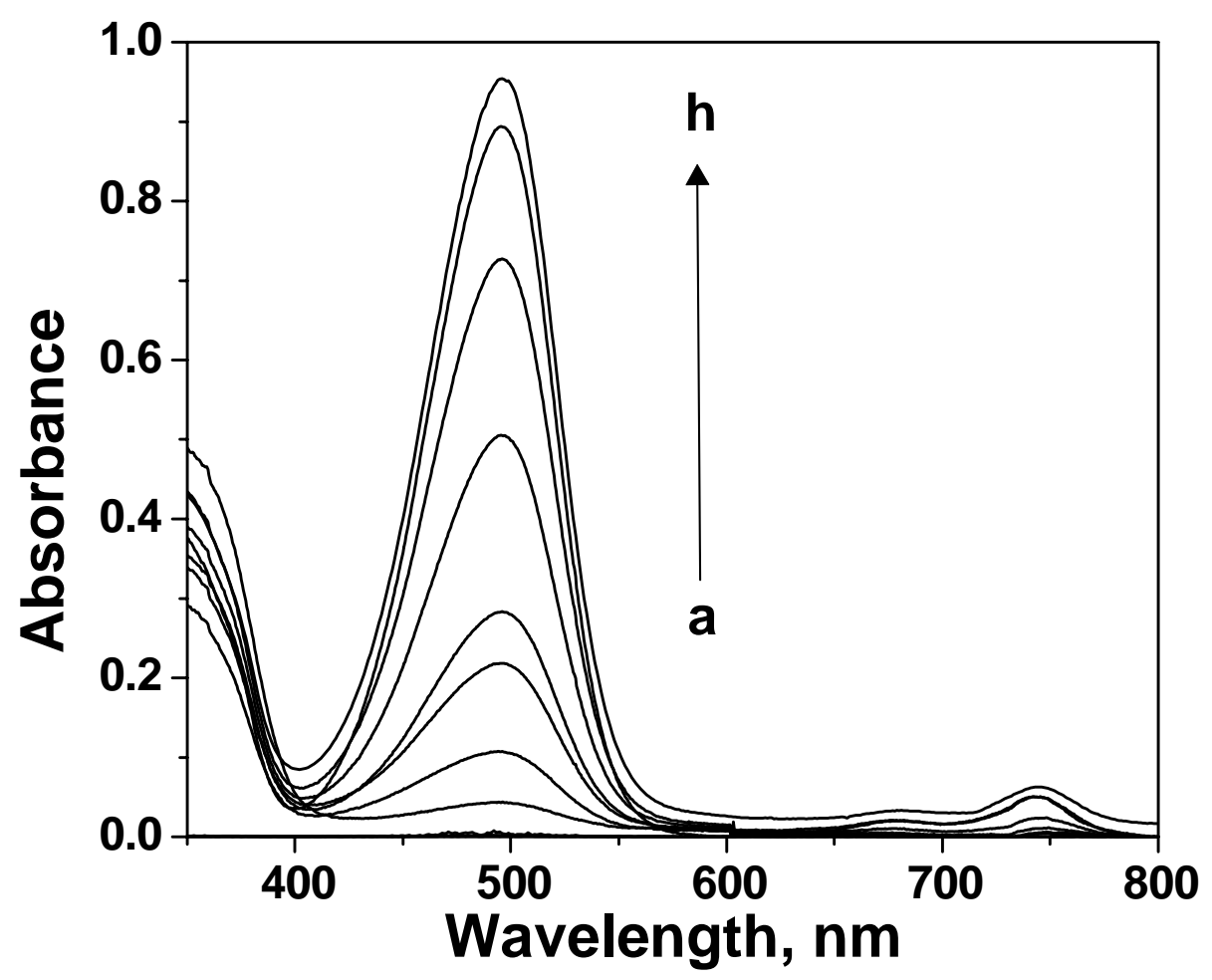

Figure S2. Change in absorption spectra obtained for the reaction between squaric acid and the quinaldinium salt 1c at various time intervals. Time (a) 1, (b) 2, (c) 3, (d) 4, (e) 6, (f) 9, (g) 12, (h) $15.5 \mathrm{~h}$. 


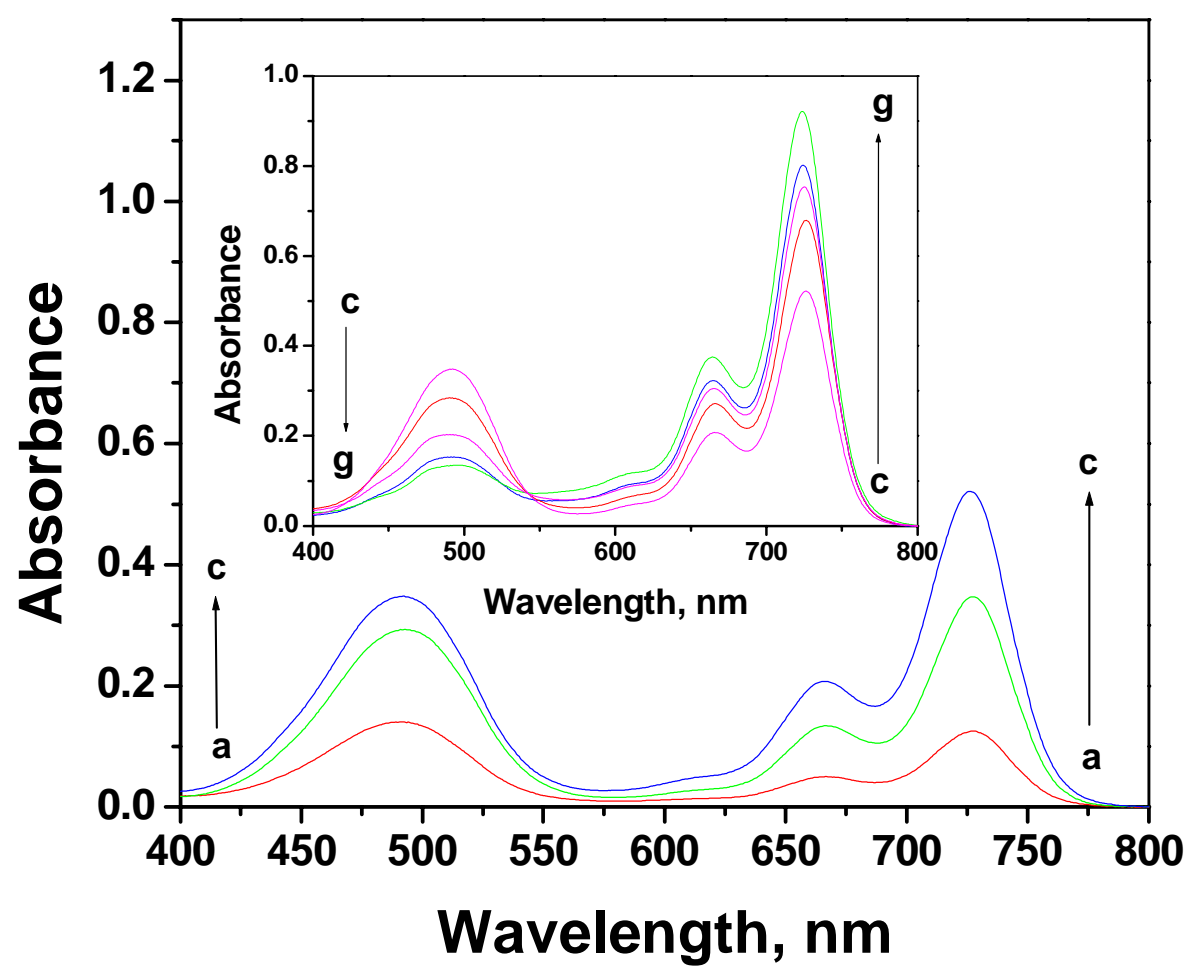

Figure S3. Change in absorption spectra obtained for the reaction between squaric acid and the quinaldinium salt 1e at various time intervals. Time (a) 1, (b) 3, (c) $5 \mathrm{~h}$. Inset shows the change in absorption spectra obtained for the same reaction after long time intervals. Time (c) 7 (d) 9 , (e) $11,(\mathbf{f}) 16,(\mathrm{~g}) 19 \mathrm{~h}$. 
(A)

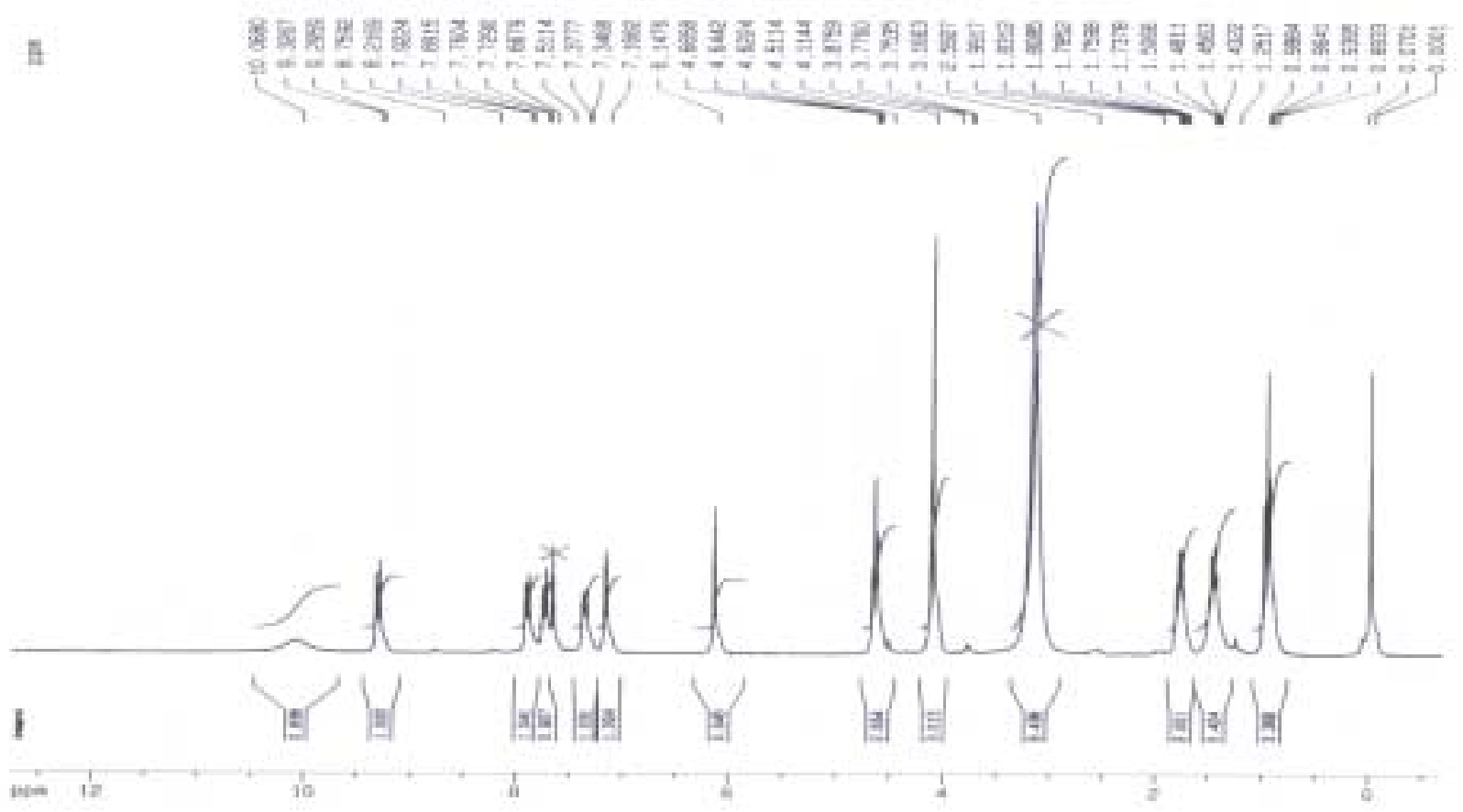

(B)
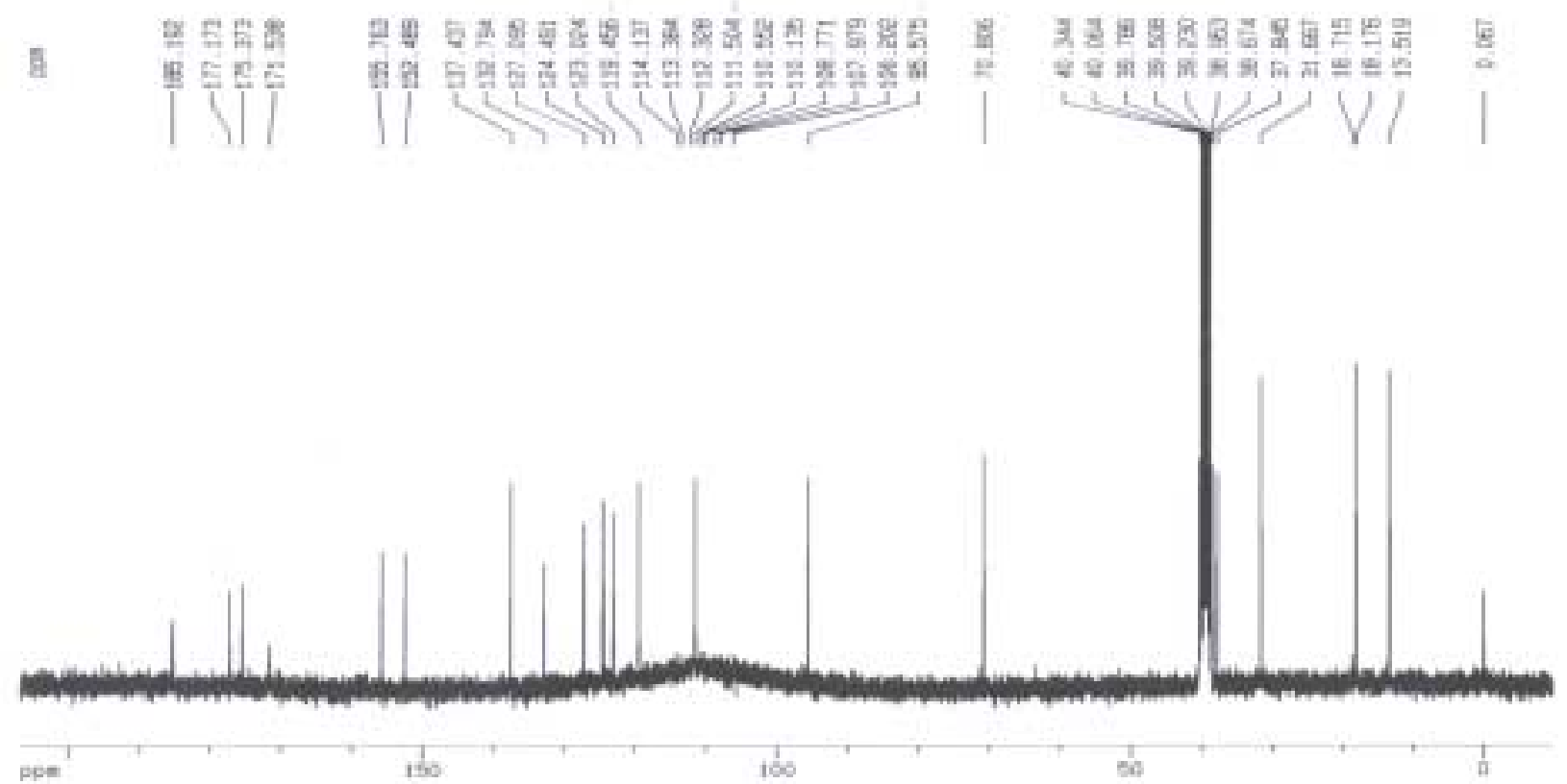

Figure S4. $\quad{ }^{1} \mathrm{H}(\mathrm{A})$ and ${ }^{13} \mathrm{C}$ NMR (B) spectra of the semisquaraine derivative 2a in DMSO- $\mathrm{d}_{6}$ : $\mathrm{CDCl}_{3}(1: 4)$ mixture and DMSO-d $\mathrm{d}_{6}$ respectively. 
(A)
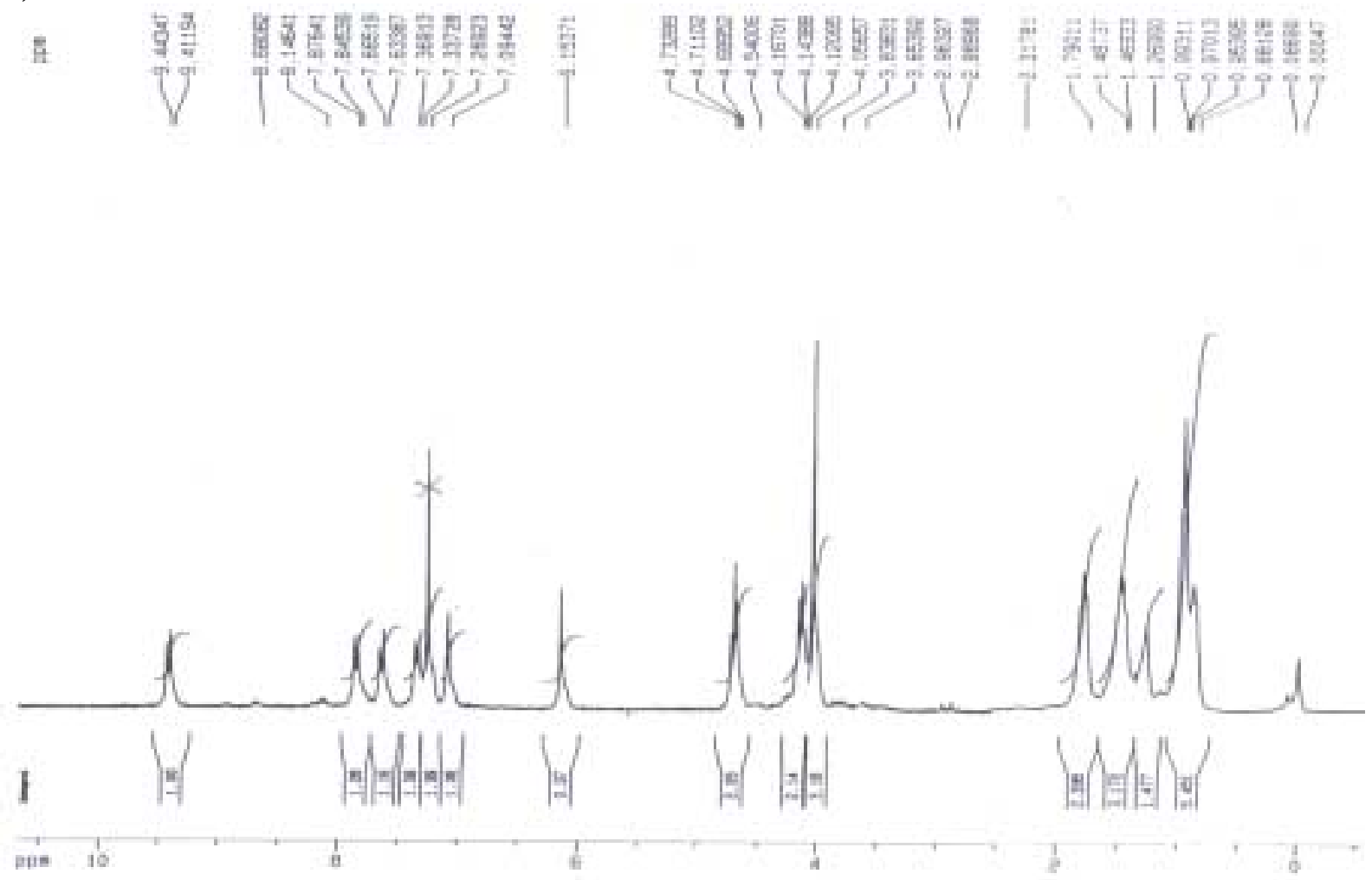

(B)

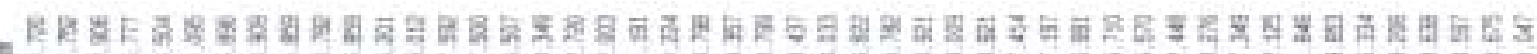

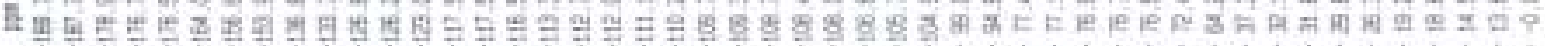

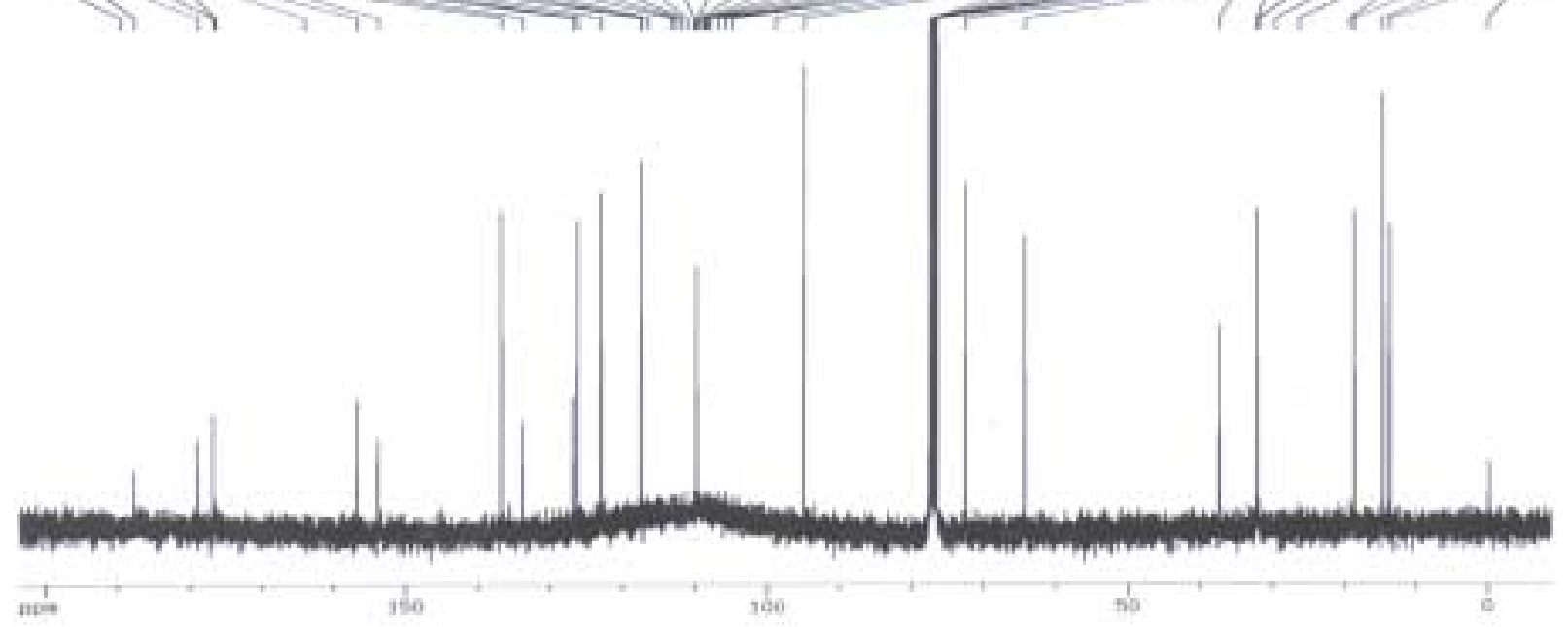

Figure S5. ${ }^{1} \mathrm{H}(\mathrm{A})$ and ${ }^{13} \mathrm{C}$ NMR (B) spectra of the semisquaraine derivative $2 \mathbf{b}$ in DMSO- $\mathrm{d}_{6}$ : $\mathrm{CDCl}_{3}(1: 4)$ mixture and DMSO-d 6 respectively. 


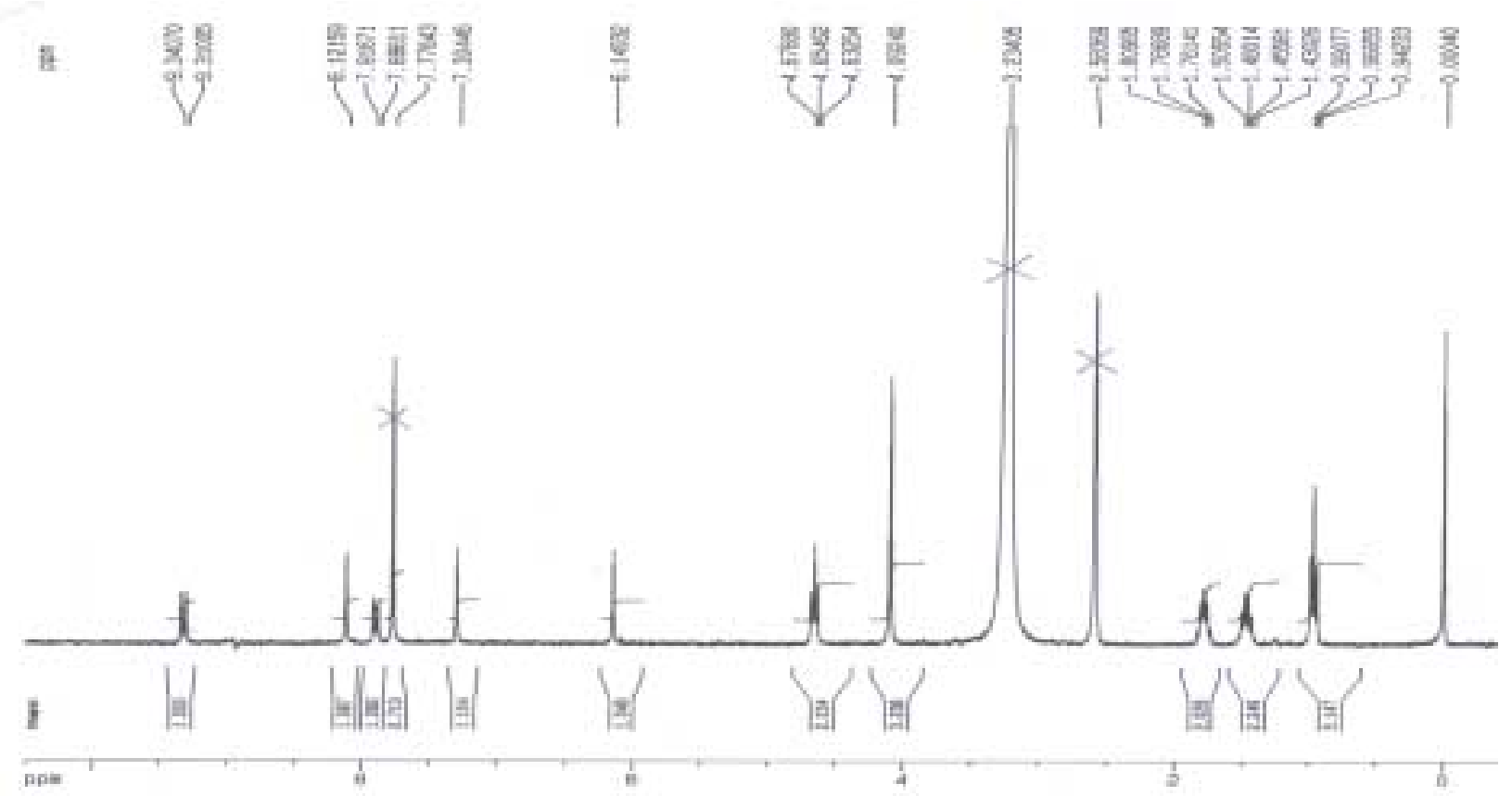

Figure S6. ${ }^{1} \mathrm{H}$ NMR spectrum of the semisquaraine derivative $\mathbf{2 c}$ in DMSO-d6.

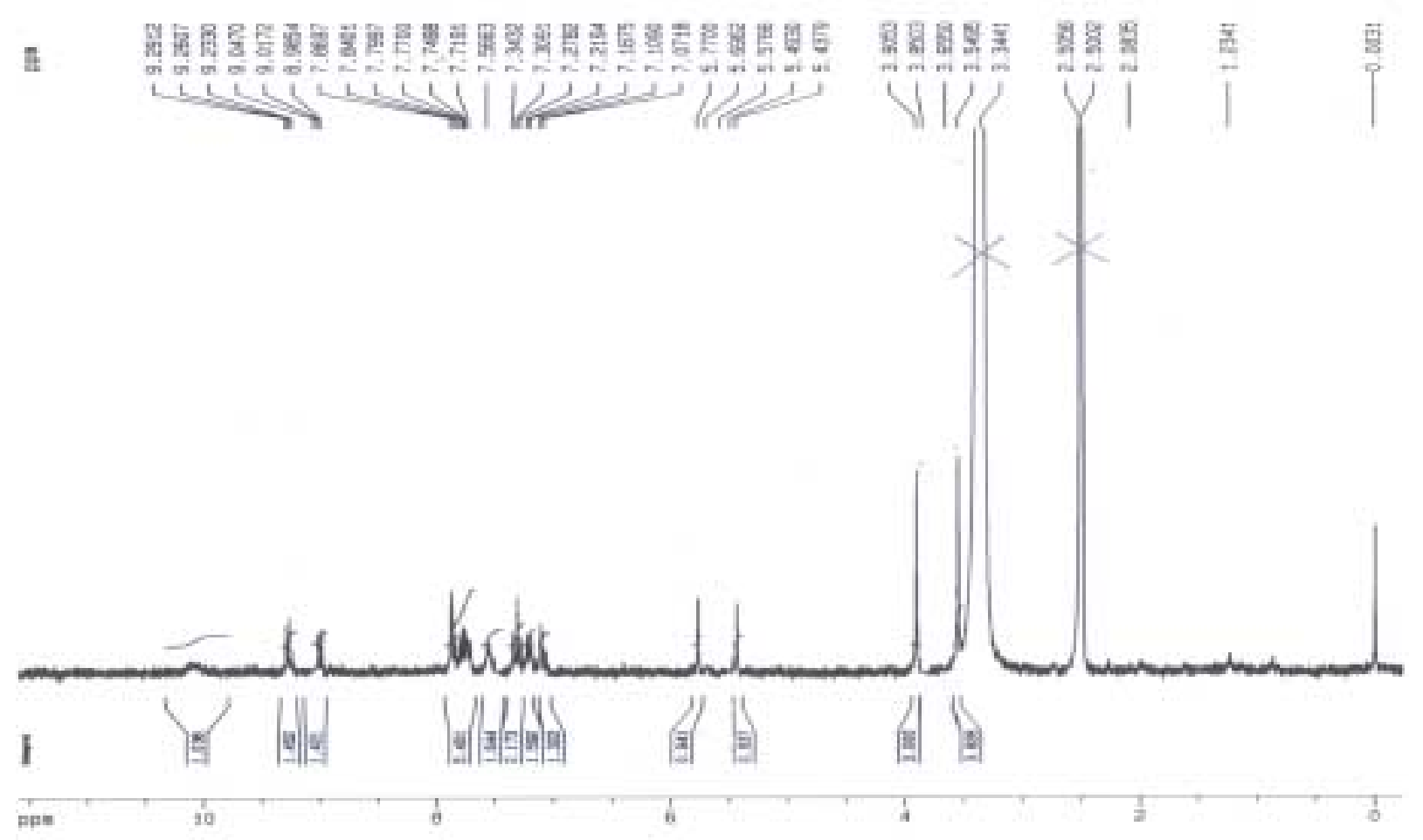

Figure S7. ${ }^{1} \mathrm{H}$ NMR spectrum of the squaraine dye 4 in DMSO-d6. 